\title{
BMJ Open Effectiveness of a lumbopelvic monitor and feedback device to change postural behaviour: a protocol for the ELF cluster randomised controlled trial
}

\author{
Daniel Cury Ribeiro, ${ }^{1}$ Stephan Milosavljevic, ${ }^{2} \mathrm{~J} \mathrm{Haxby} \mathrm{Abbott}^{3}$
}

To cite: Ribeiro DC Milosavljevic S, Abbott JH. Effectiveness of a lumbopelvic monitor and feedback device to change postural behaviour: a protocol for the ELF cluster randomised controlled trial. BMJ Open 2017;7:e015568. doi:10.1136/bmjopen-2016015568

- Prepublication history for this paper is available online. To view these files please visit the journal online (http://dx.doi.org/10.1136/ bmjopen-2016-015568).

Received 14 December 2016 Accepted 19 December 2016

CrossMark

For numbered affiliations see end of article.

Correspondence to Dr Daniel Cury Ribeiro; daniel.ribeiro@otago.ac.nz

\section{ABSTRACT}

Introduction: Low back pain (LBP) is the most common, costly and disabling musculoskeletal disorder worldwide, and is prevalent in healthcare workers. Posture is a modifiable risk factor for LBP shown to reduce the prevalence of LBP. Our feasibility research suggests that postural feedback might help healthcare workers avoid hazardous postures. The Effectiveness of Lumbopelvic Feedback (ELF) trial will investigate the extent to which postural monitor and feedback (PMF) can reduce exposure to hazardous posture associated with LBP.

Methods: This is a participant-blinded, randomised controlled trial with blocked cluster random allocation. Participants will include volunteer healthcare workers recruited from aged care institutions and hospitals. A postural monitoring and feedback device will monitor and record lumbopelvic forward bending posture, and provide audio feedback whenever the user sustains a lumbopelvic forward bending posture that exceeds predefined thresholds. The primary outcome measure will be postural behaviour (exceeding thresholds). Secondary outcome measures will be incidence of LBP, participant-reported disability and adherence. Following baseline assessment, we will randomly assign participants to 1 of 2 intervention arms: a feedback group and a no-feedback control group. We will compare between-group differences of changes in postural behaviour by using a repeated measures mixed-effect model analysis of covariance (ANCOVA) at 6 weeks. Postural behaviour baseline scores, workrelated psychosocial factors and disability scores will be input as covariates into the statistical models. We will use logistic mixed model analysis and Cox's proportional hazards for assessing the effect of a PMF on LBP incidence between groups.

Discussion: Posture is a modifiable risk factor for low back disorders. Findings from the ELF trial will inform the design of future clinical trials assessing the effectiveness of wearable technology on minimising hazardous posture during daily living activities in patients with low back disorders.

Trial registration number: ACTRN12616000449437.

\section{INTRODUCTION}

Low back pain (LBP) is a common, costly and disabling musculoskeletal disorder, ${ }^{1}$ and is prevalent in healthcare workers (eg, nurses, physiotherapists and caregivers). ${ }^{2}{ }^{3}$ Non-specific LBP (NSLBP) is a multifactorial disorder, considered to have complex interactions between mechanical, psychosocial and biological factors, which can influence the onset and maintenance of symptoms. ${ }^{4-6}$ Numerous risk factors for NSLBP are identifiable in the literature, with postural behaviour being one modifiable risk factor. ${ }^{7}$ Change to workplace posture is commonly targeted by ergonomists, clinicians and researchers during rehabilitation and prevention programmes for NSLBP. ${ }^{8}$

Given the health and care demands of an ageing population, ${ }^{10}$ it is imperative to keep the healthcare workforce physically healthy. LBP is highly prevalent in healthcare workers, and it is suggested that sustained and repetitive flexed postures are underestimated as potential causes of occupational LBP in this workforce. ${ }^{11}$ Despite the extensive amount of research on LBP, prevention and rehabilitation continues to be a challenge. ${ }^{1}$

New strategies for prevention and rehabilitation of LBP are required. The evidence supporting standard ergonomic training as an effective intervention for minimising exposure to hazardous posture at work is limited. ${ }^{12}$ Preliminary findings suggest that the use of postural feedback may help change postural behaviour, potentially contributing to prevention and management of NSLBP. ${ }^{13} 14$ The provision of real-time postural feedback during daily activities in the workplace could help healthcare workers reduce hazardous postural behaviours. ${ }^{13}$

New wearable motion sensors allow the monitoring of lumbopelvic movement 
pattern ${ }^{15}$ and the provision of postural feedback during daily life and occupational activities. ${ }^{13}{ }^{16} \quad 17$ The Spineangel (Movement Metrics, Hamilton, New Zealand) is one example of a wearable postural monitor and feedback (PMF) device. It was designed to monitor lumbopelvic movements and to provide audio feedback whenever the user adopts a specific lumbopelvic posture and sustains it for a predetermined duration. ${ }^{13}$ This type of audio feedback is characterised as intermittent feedback, ${ }^{1819}$ as it is only activated when a specific posture is exceeded. Our previous research has found this device to be reliable and valid for monitoring lumbopelvic posture in the workplace. ${ }^{15}{ }^{20}$ The device shows a withinday measurement error of $5^{\circ},{ }^{20}$ and a between-day measurement error of $8^{\circ} \cdot{ }^{15}$ Such findings support the use of this device for monitoring posture during daily life activities.

Our recent feasibility randomised controlled trial (RCT) study indicated that the use of a PMF device might reduce the time spent in a hazardous forward bent posture by healthcare workers, and that a full trial to investigate this effect would be feasible. ${ }^{13}$ The feasibility trial has also shown that the PMF device was also well received by healthcare workers, who perceived it as a useful tool to improve their working posture. ${ }^{13}$ On the basis of information gathered from our feasibility RCT ( phase II), we have designed a phase III study. ${ }^{21}$

\section{METHODS/DESIGN}

\section{Study aim}

The primary aim of this study is to assess the effectiveness of a lumbopelvic postural feedback device for changing postural behaviour in a group of healthcare workers. We hypothesise that workers exposed to auditory postural feedback will present with a reduction in time spent in flexed posture in the workplace. The secondary aim of this study is to assess the data variability when determining the effectiveness of the postural monitor device for preventing NSLBP. Identifying such variability is an essential step for a future LBP injury prevention trial.

\section{Study design}

This will be a participant-blinded, assessor-blinded, sham-controlled, parallel-group, stratified cluster RCT. The cluster RCT design will prevent treatment contamination between the intervention and control groups. ${ }^{22}{ }^{23}$ General information about the trial is presented in table 1. The University of Otago Ethics

Table 1 The WHO trial registration data set

\begin{tabular}{|c|c|}
\hline Data category & Information \\
\hline $\begin{array}{l}\text { Primary registry and trial } \\
\text { identifying number }\end{array}$ & Australian New Zealand Clinical Trial Registry (ACTRN12616000449437) \\
\hline $\begin{array}{l}\text { Date of registration in primary } \\
\text { registry }\end{array}$ & $7 / 04 / 2016$ \\
\hline Source of monetary or material & Health Research Council Emerging Researcher First Grant (15/527) \\
\hline support & Lottery Health Research (AP353007) \\
\hline Primary sponsor & University of Otago \\
\hline Contact for public queries & feedback.study@otago.ac.nz \\
\hline Contact for scientific queries & Dr Daniel Cury Ribeiro, School of Physiotherapy_University of Otago \\
\hline Public title & $\begin{array}{l}\text { Effectiveness of a lumbopelvic monitor and feedback device to change postural behaviour } \\
\text { in healthcare workers }\end{array}$ \\
\hline Scientific title & $\begin{array}{l}\text { The effectiveness of a lumbopelvic monitor and feedback device to change postural } \\
\text { behaviour in healthcare workers with or without LBP: a clustered randomised controlled } \\
\text { trial }\end{array}$ \\
\hline Country of recruitment & New Zealand \\
\hline $\begin{array}{l}\text { Health condition or problem } \\
\text { studied }\end{array}$ & LBP \\
\hline Interventions & Postural feedback and sham \\
\hline $\begin{array}{l}\text { Key inclusion and exclusion } \\
\text { criteria }\end{array}$ & $\begin{array}{l}\text { Adult healthcare workers (from } 18 \text { to } 65 \text { years), with or without LBP, presently performing } \\
\text { their regular work activities, will be included }\end{array}$ \\
\hline Study type & Interventional \\
\hline Date of first enrolment & $13 / 04 / 2016$ \\
\hline Target sample size & 108 \\
\hline Recruitment status & Recruiting \\
\hline Primary outcome & $\begin{array}{l}\text { Postural behaviour defined and expressed as the total number of times the postural } \\
\text { threshold was exceeded in a week. Postural behaviour will be measured using a } \\
\text { lumbopelvic monitor device. }\end{array}$ \\
\hline Key secondary outcome & $\begin{array}{l}\text { Presence of LBP, functional disability, Work-related psychosocial characteristics and } \\
\text { adherence. }\end{array}$ \\
\hline
\end{tabular}


Committee has approved this study protocol (H15/094). Consultation with Maori (New Zealand's indigenous people) has been undertaken and concluded. The trial is prospectively registered with the Australian and New Zealand Clinical Trials Registry (ACTRN12616000449437) and will follow the Consolidated Standards of Reporting Trials (CONSORT) statement-extension to cluster randomised trials. $^{22}$

\section{Participants and setting}

Participants will be healthcare workers recruited from aged care institutions and hospitals within the Otago and Southland provinces of New Zealand. Adult healthcare workers, with or without LBP, presently performing their regular work activities, will be included. Potential participants who work $<20$ hours/week or are unable to undertake regular work-related activities due to LBP or any other musculoskeletal disorder will be excluded from this study.

\section{Definition of postural behaviour}

For the purpose of this study, postural behaviour is the number of times per hour workers exceed a given threshold of cumulative forward flexed trunk posture. The postural threshold is the range of motion of forward bending, for a given duration of time.

In this study, postural threshold will be considered surpassed when a participant exceeds two lumbopelvic forward bending events per minute, where flexion is $\geq 45^{\circ}$, with each epoch lasting $>5 \mathrm{~s}^{13}$

\section{Equipment}

A PMF device, the Spineangel (Movement Metrics, New Zealand) will record a lumbopelvic forward bending posture. This device, the size of an electronic pager (figure 1), contains a triaxial accelerometer capable of providing an audio feedback (ie, beeping sound) whenever the user sustains a forward bending posture that exceeds the predefined postural threshold. The postural threshold is adjustable according to the range, duration

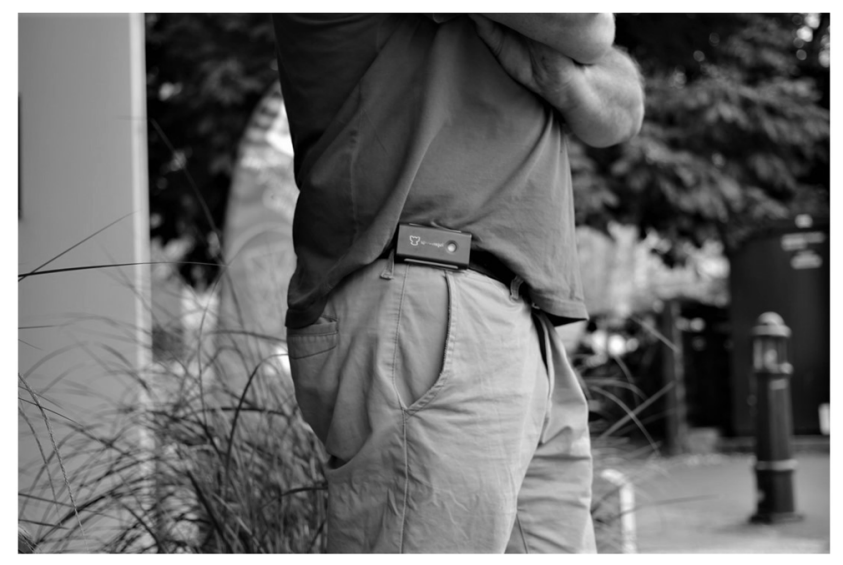

Figure 1 The postural monitor and feedback device (Spineangel). and frequency of the forward bending posture. In order to enhance the recruitment rate and optimise data collection, 82 PMF devices will be required. To enhance adherence, text messages will be sent twice a week to participants, to remind them to use the device during working hours. In addition, posters with a reminding message to participants will also be placed at the workplace.

\section{Baseline participant characteristics}

All participants will complete a self-reported questionnaire to gather demographic (age, gender, body mass, height, occupation, smoking habits), functional disability and psychosocial work characteristic data. Functional disability will be measured with the Oswestry Disability Index (ODI), while work-related psychosocial characteristics will be gathered by using the second short version of the Copenhagen Psychosocial Questionnaire (COPSOQ II) ${ }^{24}$ Since there is an association between high ODI scores and fear-avoidance behaviour, and a moderate association between work-related psychosocial factors and physical exposure, ${ }^{9}{ }^{25}$ it is possible that these variables can impact postural behaviour, and thus will be monitored and controlled for during data analysis.

\section{Outcome measures}

The primary outcome measure will be the total number of postural threshold-exceeding events per hour in a week (table 2).

Secondary outcome measures will be presence of LBP over the course of follow-up (1 year); functional disability, measured with the Oswestry Questionnaire and expressed as a percentage of total possible score; and adherence assessed as the number of days participants work with the lumbopelvic monitor and feedback device, expressed as a percentage of total working days of each week of data collection.

\section{Outcomes assessment}

Data will be collected over a 6-week period and then at follow-up time points (figure 2). During the 1st and 6th weeks, we will collect baseline and postintervention measurements.

\section{Baseline postural behaviour assessment}

Baseline measurements will be gathered over 1 week, prior to starting intervention. During this period, the lumbopelvic motion monitor will only record data; no postural audio feedback will be provided to participants.

\section{Follow-up assessments}

Follow-up will occur at the 6th week of data collection, and at 1, 3, 6 and 12 months after the 6 th week. At the end of the 6th week, and follow-up time points $(1,3,6$ and 12 months), participants will complete a questionnaire on current functional status (Oswestry Questionnaire) and will wear the lumbopelvic motion 
Table 2 Overview of outcome measures and time points

\begin{tabular}{|c|c|c|}
\hline Variable & Data resource and instrument & Time points (months) \\
\hline \multicolumn{3}{|l|}{ Primary outcome } \\
\hline Postural behaviour & $\begin{array}{l}\text { Measured with a postural monitor device, expressed as the } \\
\text { total number of times the postural threshold was exceeded in a } \\
\text { week }\end{array}$ & $\begin{array}{l}\text { Baseline, week } 6 \text {; and } 1,3,6 \\
\text { and } 12 \text { months after baseline }\end{array}$ \\
\hline \multicolumn{3}{|l|}{ Secondary outcomes } \\
\hline Presence of LBP & Measured with using the Delphi DOLBaPP Questionnaire & $\begin{array}{l}\text { Baseline, week } 6 \text {; and } 1,3,6 \\
\text { and } 12 \text { months after baseline }\end{array}$ \\
\hline $\begin{array}{l}\text { Work-related } \\
\text { psychosocial } \\
\text { characteristics }\end{array}$ & Measured with COPSOQ II & Baseline \\
\hline Adherence & $\begin{array}{l}\text { Assessed as the number of days participants worked with the } \\
\text { lumbopelvic monitor and feedback device, and will be } \\
\text { expressed as a percentage of total working days of each week } \\
\text { of data collection. }\end{array}$ & $\begin{array}{l}\text { Baseline, weeks } 1-6 \text {; and } 1,3 \text {, } \\
6 \text { and } 12 \text { months after baseline }\end{array}$ \\
\hline Age & Self-administered questionnaire & Baseline \\
\hline Height & Self-administered questionnaire & Baseline \\
\hline Weight & Self-administered questionnaire & Baseline \\
\hline
\end{tabular}

monitor device daily for 1 week in order to monitor and record postural behaviour.

\section{Intervention}

The intervention period consists of 4 weeks (weeks 2-5, inclusive), and participants will either receive or not receive postural audio feedback according to randomised allocation into one of the following groups: sham group (SG), feedback group (FG). On the 1st day of the intervention period, participants from both groups will receive written and verbal instructions for using the postural device, and will be instructed to rearrange their posture (as per health and safety guidelines) in such a way that the device stops beeping. However, only participants in the FG will receive the audio feedback if the postural threshold is exceeded.

\section{Randomisation and allocation concealment}

Randomisation will be stratified by cluster size into two strata (1-9 participants; or more than 10 participants per centre). Within each stratum, centres will be randomly assigned to one of the two groups (SG or FG). The randomisation schedule will use Randomization. com, a free online randomisation program, by an investigator (JHA) not involved in recruitment, assessment or statistical analysis. To ensure allocation concealment, the investigator (JHA) will hold the randomisation schedule, and will sequentially provide, by stratum, notification of group allocation to the field coordinator (JT) as each new site (cluster) is recruited. The expected flow of participants through the trial is illustrated in figure 3 (as per Consolidated Standards of Reporting Trials
(CONSORT) statement) and figure 4 (a graphical approach that has been recently developed and recommended for enhancing clarity and quality of reporting of cluster trials). ${ }^{26}$

1. Cluster identification: The research team will identify aged care institutions and hospitals within the Otago and Southland provinces of New Zealand.

2. Cluster recruitment: Aged care institutions and hospitals will be invited by post. The field coordinator visits the aged care institutions and hospitals that express intention to participate in the study, and will explain the study in detail to the manager (face to face or by telephone).

3. Participant Identification: The field coordinator visits the aged care institutions and hospitals that express intention to participate in the study, and now explains the study in detail to healthcare workers.

4. Cluster recruitment: Those healthcare workers who are willing to take part in the study sign a written consent form and their contact details are gathered by the field coordinator. Not all healthcare workers from the aged care institution need to consent to participate for the institution to be included in the study.

5. Baseline assessment: Participants complete a selfreported questionnaire to gather demographic (age, gender, body mass, height, occupation, smoking habits), functional disability and psychosocial work characteristic data. Postural behaviour is recorded during the 1st week of data collection.

6. Randomisation: The randomisation schedule will use a free online randomisation program (Randomization. com) by an investigator (JHA) not involved in 


\begin{tabular}{|c|c|c|c|c|c|c|c|c|c|c|c|c|}
\hline & \multicolumn{12}{|c|}{ Study Period } \\
\hline & Enrolment & Allocation & & & & & & t all & cati & & & \\
\hline Time point & $-\mathrm{t} 1$ & 0 & $\mathrm{t} 1$ & $\mathrm{t} 2$ & t3 & t4 & $\mathrm{t} 5$ & t6 & t7 & t8 & t9 & $\mathrm{t} 10$ \\
\hline Enrolment & & & & & & & & & & & & \\
\hline Eligibility screen & $\mathrm{X}$ & & & & & & & & & & & \\
\hline Informed consent & $\mathrm{X}$ & & & & & & & & & & & \\
\hline Allocation & & & & & & & & & & & & \\
\hline & & & & & & & & & & & & \\
\hline Intervention & & & & & & & & & & & & \\
\hline Control Group & & & & & & & & & $\rightarrow$ & & & \\
\hline Feedback Group & & & & & & & & & $\Rightarrow$ & & & \\
\hline & & & & & & & & & & & & \\
\hline Assessments & & & & & & & & & & & & \\
\hline $\begin{array}{l}\text { Baseline } \\
\text { Demographic } \\
\text { information }\end{array}$ & $\mathrm{x}$ & & & & & & & & & & & \\
\hline $\begin{array}{l}\text { Covariates: } \\
\text { Age }\end{array}$ & & & $\mathrm{X}$ & & & & & & & & & \\
\hline Height & & & $\mathrm{X}$ & & & & & & & & & \\
\hline Weight & & & $\mathrm{X}$ & & & & & & & & & \\
\hline $\begin{array}{l}\text { Work-related } \\
\text { psychosocial } \\
\text { characteristics }\end{array}$ & & & $\mathrm{X}$ & & & & & & & & & \\
\hline $\begin{array}{l}\text { Primary, and } \\
\text { secondary } \\
\text { outcome } \\
\text { measures: }\end{array}$ & & & & & & & & & & & & \\
\hline $\begin{array}{l}\text { Postural } \\
\text { behaviour }\end{array}$ & & & $\mathrm{X}$ & $\mathrm{X}$ & $\mathrm{X}$ & $\mathrm{X}$ & $\mathrm{X}$ & $\mathrm{X}$ & $\mathrm{X}$ & $\mathrm{X}$ & $\mathrm{x}$ & $\mathrm{X}$ \\
\hline Presence of LBP & & & $\mathrm{X}$ & & & & & $\mathrm{X}$ & $\mathrm{X}$ & $\mathrm{X}$ & $\mathrm{X}$ & $\mathrm{X}$ \\
\hline $\begin{array}{l}\text { Functional } \\
\text { Disability }\end{array}$ & & & $\mathrm{X}$ & & & & & $\mathrm{X}$ & $\mathrm{X}$ & $\mathrm{X}$ & $\mathrm{X}$ & $\mathrm{X}$ \\
\hline Adherence & & & $\mathrm{X}$ & $\mathrm{X}$ & $\mathrm{X}$ & $\mathrm{X}$ & $\mathrm{X}$ & $\mathrm{X}$ & $\mathrm{X}$ & $\mathrm{X}$ & $\mathrm{X}$ & $\mathrm{X}$ \\
\hline
\end{tabular}

Figure 2 Schedule for enrolment and intervention per cluster. t1: baseline; t2-t5: intervention period; t6: 6 weeks after baseline; t7: 1 month after baseline; t8: 3 months after baseline; t9: 6 months after baseline; t10: 12 months after baseline. LBP, low back pain.

recruitment, assessment or statistical analysis. To ensure allocation concealment, the investigator (JHA) will hold the randomisation schedule, and will sequentially provide, by stratum, notification of group allocation to the field coordinator (JT) as each new site (cluster) is recruited.

7. The field coordinator will not be blinded to group allocation and will be responsible for downloading the data from the postural monitor feedback device. Risk of bias is minimal as the postural monitor and feedback (PMF) device will record the primary outcome and secondary outcomes are participant self-reported questionnaires. Participants will be blinded to group allocation; our feasibility study indicated that participant blinding was successfully maintained. (A) Feedback intervention: Participants will receive postural audio feedback whenever the postural threshold is exceeded. (B) Sham intervention: Participants will not receive any postural audio feedback, and the postural monitor feedback will just record postural behaviour.
8. Outcome assessment: Follow-up will occur at the 6th week of data collection, and at 1, 3, 6 and 12 months after the 6 th week. At the end of the 6 th week, and follow-up time points (1, 3, 6 and 12 months), participants will complete a questionnaire on current functional status (Oswestry Questionnaire) and will wear the lumbopelvic motion monitor device daily for 1 week in order to monitor and record postural behaviour. The researcher responsible for statistical analysis will be blinded to group allocation until data analysis is complete.

\section{Blinding}

Participants will be blinded to group allocation. The researcher responsible for statistical analysis (DCR) will also be blinded to group allocation until data analysis is complete. The field coordinator (JT) will be responsible for setting the PMF device and delivering the devices to workers. The field coordinator will be aware of group allocation and will be responsible for 


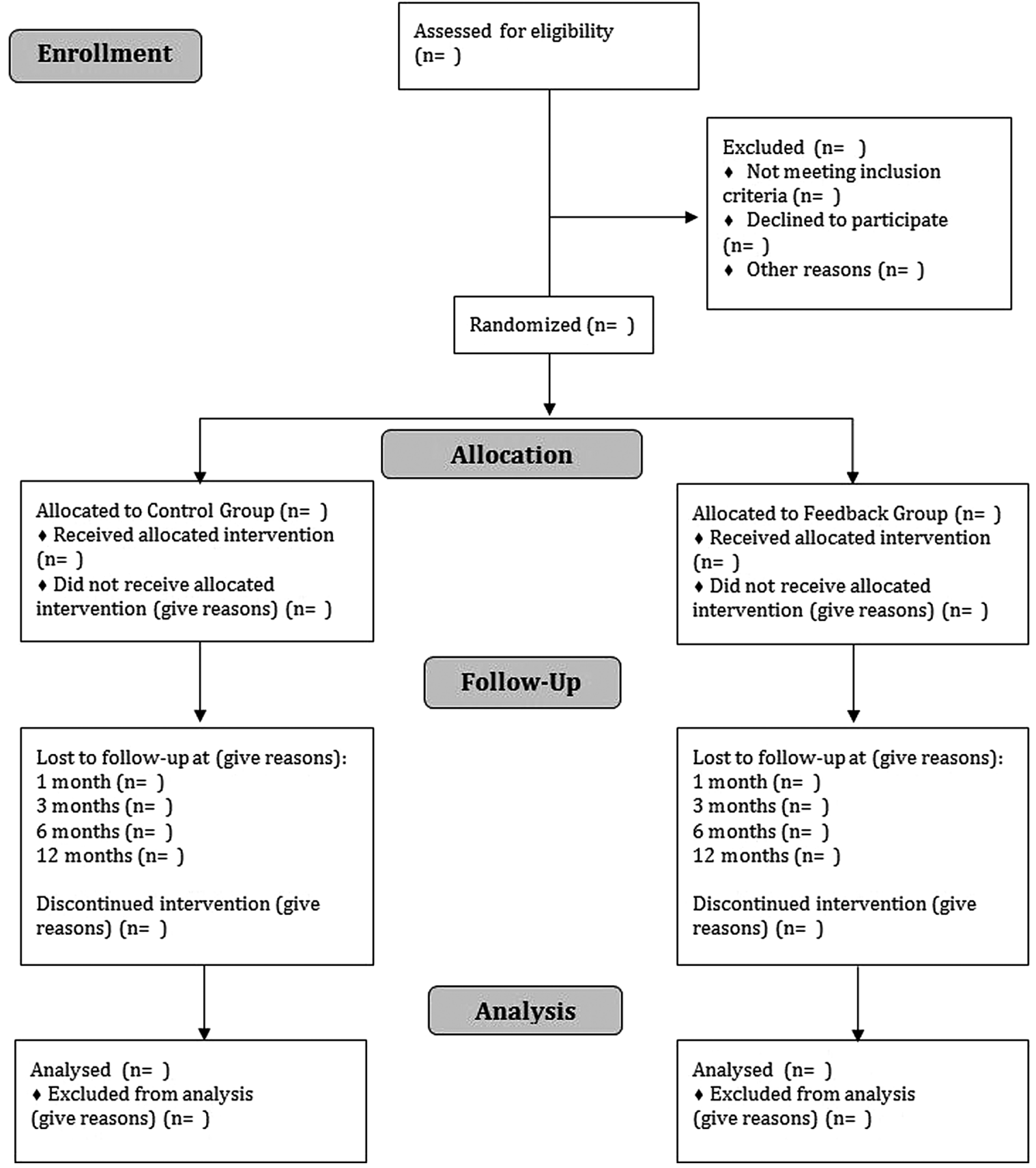

Figure 3 Diagram of participant flow at the Effectiveness of Lumbopelvic Feedback (ELF) trial.

downloading the data from the postural monitor feedback device. Risk of bias is minimal as the PMF device will record the primary outcome and secondary outcomes are participant self-reported. Our feasibility study indicates that participant blinding can be successfully maintained. ${ }^{13}$

\section{Sham group}

Participants allocated to the SG will not receive postural audio feedback and the PMF device will be set to monitor and record postural behaviour only, with feedback capability switched off. The participant cannot alter this setting.

\section{Feedback group}

The FG will receive postural audio feedback whenever the postural threshold is exceeded, during the 4 weeks of intervention. The postural threshold is set on the PMF device by adjusting the range of motion limit to $45^{\circ}$ of lumbopelvic forward bending, frequency of lumbopelvic forward-bending (maximum of two events exceeding the range of motion threshold per minute), and duration of sustained bending set at $5 \mathrm{~s} .{ }^{13} 20$

\section{Sample size estimation}

The estimated sample size is based on published recommendations by Rutterford $e t a l^{27}$ for determining sample size in cluster randomised trials. This requires initially 
Figure 4 Timeline cluster diagram for the Effectiveness of Lumbopelvic Feedback (ELF) trial.

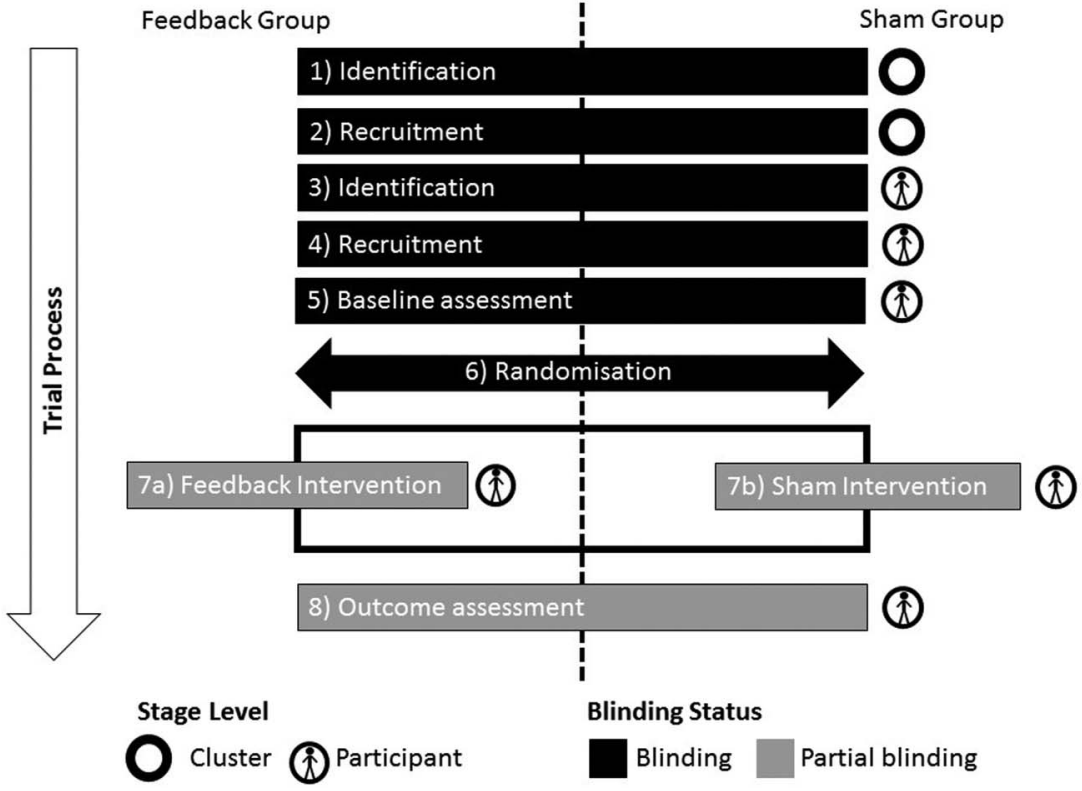

estimating the sample size for a standard, single-site RCT. This was calculated using the 'pwr' package in R Statistical Software. ${ }^{28}$ Assuming an effect size of 0.6 (based on our published feasibility RCT) ${ }^{13} \alpha$ of 0.05 , and power of $80 \%$ as input data for sample size calculation, a two-arm RCT, with an allocation ratio of 1:1, and a one-tailed test appropriate to a superiority hypothesis, we have calculated that 35 subjects per group is the minimum required to achieve a statistical significance level of 0.05 with a power of 0.80 . Assuming a $15 \%$ drop-out rate, a minimum of 40 participants per group would be required for a standard, single-site RCT.

The sample size for the cluster RCT was estimated in two steps-first by calculating the design effect (DE), using the following formula:

$$
\mathrm{DE}=1+\left[\left(1+\mathrm{CV}^{2}\right) \times \overline{\mathrm{m}}-1\right] \rho
$$

Where:

$\mathrm{CV}=$ coefficient of variation of cluster size;

$\overline{\mathrm{m}}=$ mean cluster size;

$\rho=$ intracluster correlation coefficient.

Then, following calculation of the DE, the sample size for the cluster RCT was determined using equation (2): ${ }^{29}$

Where:

$$
\mathrm{SS}_{\text {Clustered }}=\mathrm{SS}_{\mathrm{RCT}} \times \mathrm{DE}
$$

$\mathrm{SS}_{\text {Clustered }}=$ sample size for cluster RCT;

$\mathrm{SS}_{\mathrm{RCT}}=$ sample size for standard RCT;

$\mathrm{DE}=$ design effect.

Equation (1) allows estimation of sample size for a cluster RCT with unequal cluster sizes. ${ }^{29}$ The CV is estimated by dividing the SD of the cluster size by the estimated mean cluster size. ${ }^{29}$ The SD of the cluster size can be estimated by anticipating the likely range of cluster sizes and dividing it by four. ${ }^{29}$ For the purpose of this study, the estimated maximum cluster size is 15 , and the estimated minimum cluster size is 5, giving a likely cluster size range of 10 . The mean cluster size is assumed to be 7 . The intracluster correlation coefficient ( $\rho)$ was calculated based on data from the feasibility RCT, and was equal to 0.05. Based on equation (1) and the values above described, the DE equalled 1.33. Therefore, using equation (2), the estimated sample size for the cluster RCT was a minimum of 108 participants, with 15 clusters ( $\sim 7$ participants per cluster).

The method we used (equation (2)) for estimating the DE tends to overestimate the sample size, and less conservative methods have been described in the literature. $^{27}{ }^{30}$ We opted for a conservative estimation of the DE to counteract the possibility that our feasibility RCT may have inflated the true effect size for the intervention. ${ }^{31}$

\section{Statistical analysis}

All statistical analyses will be performed using $\mathrm{R}$ software (R_Core_Team. R: A language and environment for statistical computing. $\mathrm{R}$ Foundation for Statistical Computing, Vienna, Austria. ISBN 3-900051-07-0, URL http://www.R-project.org. Secondary R: A language and environment for statistical computing. R Foundation for Statistical Computing, Vienna, Austria. ISBN 3-90005107-0, URL http://www.R-project.org. 2008. http://www. R-project.org). Intention-to-treat analysis will estimate the effect of feedback provision on postural behaviour. For all statistical analysis, $\alpha$ will be set at 0.05 . Missing data will be dealt with by using maximum likelihood estimation. $^{32}$

\section{Analysis for study aim 1}

Since baseline imbalances reduce statistical power, and the use of cluster randomisation increases risk of baseline imbalances, ${ }^{27}$ we will compensate for power loss by increasing precision through adjustment for covariates 
(ie, baseline demographic data; functional disability measured with the ODI; and psychosocial factors measured with the COPSOQ II) in the statistical analysis. ${ }^{27} 30$ All statistical analyses will use individual-level data.

We will compare between-group differences for changes in postural behaviour by using a repeated measures mixed-effect model analysis of covariance (ANCOVA) at 6 weeks. We will also report within-group changes by repeated measures mixed-effect model analysis of variance (ANOVA). The difference between the two models is that the 'time point' factor will include baseline measurements for the within-group comparison. This will allow us to assess the within-group immediate changes in postural behaviour at the 6th week. For the between-group comparison, baseline measurements will be a covariate controlling for baseline imbalances and removed from the 'time point' factor.

For the primary outcome of between-group difference, postural behaviour data (as measured by the PMF device) will be used as the dependent variable, with participants and clusters considered as random effects, and follow-up time points (6th week, 1, 3, 6 and 12 months). Baseline postural behaviour, work-related psychosocial factors and ODI scores were considered as fixed effects, to control for baseline imbalances. 'Time point $x$ intervention' interaction will be included in the model. Significant interactions and main effects will be followed up with planned contrasts, with $\alpha$ adjusted for multiple comparisons. $^{33}$

Within-group changes will consider postural behaviour data (as measured by the PMF device) as the dependent variable; participants and clusters will be considered as random effects; and follow-up time points (baseline, 6th week, 1, 3, 6 and 12 months), baseline postural behaviour, work-related psychosocial factors and ODI scores will be considered as fixed effects. Significant interactions and main effects will be followed up with planned contrasts, with $\alpha$ adjusted for multiple comparisons. ${ }^{33}$

\section{Analysis for study aim 2}

Research question 2 is a secondary analysis; the findings related to this research question will be subsequently submitted for publication as a separate manuscript. The primary outcome measures for research question 2 are LBP disability scores and presence of LBP.

The effect of postural feedback intervention on LBP disability scores will be assessed by using LBP disability scores (ODI score) as the dependent variable. The statistical analyses will be structured in the same way as described above.

A discrete time to event (survival) analysis will be used to assess the time to LBP resolution and time to developing LBP using logistic regression. ${ }^{34}$ We will consider the effects of baseline measurements (ie, age, body mass index, smoking habits, postural pattern at baseline, psychosocial factors and Oswestry scores) and intervention group (ie, control group or FG) on time to LBP resolution for those who have LBP, and time to developing
LBP on participants who are symptom-free at baseline. Unadjusted survival analyses will use the demographic and intervention effects on the resolution or development of LBP. If numbers permit, we will also use adjusted models. Kaplan-Meier analyses will assess 'discrete time survival' between groups for both time to LBP resolution and time to developing LBP. Survival curves will be constructed based on follow-up timepoints, while proportional hazards models will be used to assess the HR for each intervention group (ie, control group and FG), ${ }^{34} 35$ and the HRs and their respective 95\% CIs and $\mathrm{p}$ values will be reported.

\section{Protocol amendments}

We will report any protocol change likely to impact on the outcomes of the study that may potentially benefit participants or impact on participant safety (eg, study objectives and/or design changes, healthcare work population, sample size, study procedures or significant administrative changes).

\section{Dissemination policy}

The principal investigator (DCR) will be responsible for drafting and submitting the results of this trial for publication. We will adhere to the International Committee of Medical Journal Editors criteria for authorship. ${ }^{36}$ The funder will not be involved with the analysis or interpretation of the results, or the decision to submit or publish.

\section{Trial monitoring}

The Health Research Council (HRC) Data Monitoring Core Committee (New Zealand) categorised this trial as low risk, and concluded that an independent Data Monitoring Committee was not necessary. The HRC Data Monitoring Core Committee recommended that an internal monitoring process would be sufficient to oversee and monitor this trial. Therefore, the Data Monitoring Committee from the 'Centre for Health, Activity and Rehabilitation Research' (School of Physiotherapy-University of Otago) will have oversight of the trial. The research team has opted not to undertake interim analysis.

\section{Adverse event management}

The risk of any adverse event is minimal. In case of an adverse event, the primary investigator will consult with the internal Data Monitoring Committee (Centre for Health, Activity and Rehabilitation Research-University of Otago) to determine whether it is necessary to report the adverse event to the trial sponsor, and Ethics Committee. In the event that more than one serious adverse event of any kind occurs that was avoidable and related to the intervention, we will suspend the trial. If the cause of the events cannot be determined or remediated, and is plausibly related to the intervention, we will terminate the trial. 


\section{DISCUSSION}

The Effectiveness of Lumbopelvic Feedback (ELF) trial will be one of the first trials to assess whether a PMF device can change postural behaviour in an at-risk group of healthcare workers.

Posture is one modifiable risk factor for the incidence or persistence of low back disorders. Findings from this study will inform the design of future clinical trials assessing the effectiveness of wearable technology for minimising hazardous posture during daily living activities, and reducing the incidence or persistence of low back disorders.

Cluster trials are prone to a higher risk of recruitment, performance and detection bias. ${ }^{26}$ We have adopted some strategies to minimise the risk bias in this trial. To reduce the risk of recruitment bias, we will identify and recruit participants before the randomisation. The field coordinator will provide the same verbal and written instructions to participants during the intervention period, decreasing the chance of performance bias. The chance of detection bias is minimal as the primary outcome measure (postural behaviour) is measured by the postural monitor device and secondary outcome measure is obtained through participant-reported questionnaires.

\section{DECLARATIONS}

\section{Consent to participate}

Aged care institutions will provide consent for the research team to invite healthcare workers to take part in the study. Individual consent will then be obtained to enrol participants in the trial.

\section{Availability of data and material}

Participant data will be stored on a secure local server. We will use a unique identification number on follow-up questionnaires. For the protection of participants' privacy, identifying information will be stored separately, and deleted following the conclusion of the trial. No identifying information will be shared or reported.

\section{Confidentiality}

Only the research team will have access to personal information. The group mean data will be presented in the final research report, publications and conference presentations. This will protect confidentiality before, during and after the trial.

\author{
Author affiliations \\ ${ }^{1}$ Centre for Health, Activity and Rehabilitation Research, School of \\ Physiotherapy-University of Otago, Dunedin, Otago, New Zealand \\ ${ }^{2}$ School of Physical Therapy, College of Medicine, University of Saskatchewan, \\ Saskatoon, Saskatchewan, Canada \\ ${ }^{3}$ Department of Surgical Sciences, Centre for Musculoskeletal Outcomes \\ Research, Dunedin School of Medicine, University of Otago, Dunedin, New \\ Zealand
}

Twitter Follow Daniel Ribeiro @danielcr
Acknowledgements The authors thank Mr Andrew Gray for statistical advice, and thank the financial support from the Centre for Health, Activity and Rehabilitation Research-School of Physiotherapy-University of Otago, Lottery Health Research-New Zealand, and Health Research Council New Zealand.

Contributors DCR conceived of the project, was responsible for the design of the trial, and is the guarantor. SM and JHA contributed to the design of the trial. JHA developed the research programme line of enquiry. DCR led efforts for securing funding with contributions and guidance from SM and JHA. All authors revised and approved the protocol for the study. All authors revised the manuscript for important content and approved the final version.

Funding This work was supported by the Health Research Council New Zealand (15/527) and Lottery Health Research (AP353007). The trial sponsor is the University of Otago. The trial started in April 2016, and is funded until 2018.

\section{Competing interests None declared.}

Ethics approval This study protocol is approved by the University of Otago Ethics Committee (H15/094).

Provenance and peer review Not commissioned; peer reviewed for ethical and funding approval prior to submission.

Data sharing statement Data from this study will not be made publicly available. If other researchers are interested in the original data, this may be made available upon request to the principal investigator.

Open Access This is an Open Access article distributed in accordance with the Creative Commons Attribution Non Commercial (CC BY-NC 4.0) license, which permits others to distribute, remix, adapt, build upon this work noncommercially, and license their derivative works on different terms, provided the original work is properly cited and the use is non-commercial. See: http:// creativecommons.org/licenses/by-nc/4.0/

\section{REFERENCES}

1. Hoy D, March L, Brooks $P$, et al. The global burden of low back pain: estimates from the Global Burden of Disease 2010 study. Ann Rheum Dis 2014;73:968-74.

2. Harcombe $H$, McBride D, Derrett $S$, et al. Prevalence and impact of musculoskeletal disorders in New Zealand nurses, postal workers and office workers. Aust N Z J Public Health 2009;33:437-41.

3. Falavigna A, Teles AR, Mazzocchin T, et al. Increased prevalence of low back pain among physiotherapy students compared to medical students. Eur Spine J 2011;20:500-5.

4. Waddell G. The biopsychosocial model. In: Waddell G, ed. The back pain revolution. Edinburgh, New York: Churchill Livingstone, 2004: xiii, $475 \mathrm{p}$.

5. Hoogendoorn WE, Bongers PM, De Vet HCW, et al. Psychosocial work characteristics and psychological strain in relation to low-back pain. Scand J Work Environ Health 2001;27:258-67.

6. Mitchell T, O'Sullivan PB, Smith A, et al. Biopsychosocial factors are associated with low back pain in female nursing students: a cross-sectional study. Int J Nurs Stud 2009;46:678-88.

7. Coenen $\mathrm{P}$, Kingma I, Boot CR, et al. Cumulative mechanical low-back load at work is a determinant of low-back pain. Occup Environ Med 2014;71:332-7.

8. Caneiro JP, Labie C, Sulley E, et al. An exploration of familia associations of two movement pattern-derived subgroups of chronic disabling low back pain; a cross-sectional cohort study. Man Ther 2016;22:202-10.

9. Vandergrift JL, Gold JE, Hanlon A, et al. Physical and psychosocial ergonomic risk factors for low back pain in automobile manufacturing workers. Occup Environ Med 2012;69:29-34.

10. Amalberti R, Nicklin W, Braithwaite J. Preparing national health systems to cope with the impending tsunami of ageing and its associated complexities: towards more sustainable health care. Int J Qual Health Care 2016;28:412-14.

11. Freitag S, Fincke-Junod I, Seddouki R, et al. Frequent bending-an underestimated burden in nursing professions. Ann Occup Hyg 2012;56:697-707.

12. Burton AK, Balague F, Cardon G, et al., COST B13 Working Group on Guidelines for Prevention in Low Back Pain. Chapter 2. European guidelines for prevention in low back pain: November 2004. Eur Spine J 2006;15(Suppl 2):S136-68. 
13. Ribeiro DC, Sole G, Abbott JH, et al. The effectiveness of a lumbopelvic monitor and feedback device to change postura behavior: a feasibility randomized controlled trial. J Orthop Sports Phys Ther 2014:44:702-11.

14. O'Sullivan K, O'Sullivan L, O'Sullivan $P$, et al. Investigating the effect of real-time spinal postural biofeedback on seated discomfort in people with non-specific chronic low back pain. Ergonomics 2013;56:1315-25.

15. Ribeiro DC, Sole G, Abbott JH, et al. Validity and reliability of the Spineangel lumbo-pelvic postural monitor. Ergonomics 2013;56:977-91.

16. O'Sullivan K, O'Sullivan L, Campbell A, et al. Towards monitoring lumbo-pelvic posture in real-life situations: concurrent validity of a novel posture monitor and a traditional laboratory-based motion analysis system. Man Ther 2012;17:77-83.

17. Kent $P$, Laird $R$, Haines $T$. The effect of changing movement and posture using motion-sensor biofeedback, versus guidelines-based care, on the clinical outcomes of people with sub-acute or chronic low back pain-a multicentre, cluster-randomised, placebo-controlled, pilot trial. BMC Musculoskelet Disord 2015;16:131.

18. Ribeiro DC, Sole G, Abbott JH, et al. Extrinsic feedback and management of low back pain: a critical review of the literature. Man Ther 2011;16:231-9.

19. Ribeiro DC, Sole G, Abbott JH, et al. A rationale for the provision of extrinsic feedback towards management of low back pain. Man The 2011;16:301-5.

20. Ribeiro DC, Sole G, Abbott JH, et al. Cumulative postural exposure measured by a novel device: a preliminary study. Ergonomics 2011;54:858-65.

21. Stanley K. Design of randomized controlled trials. Circulation 2007;115:1164-9.

22. Campbell MK, Piaggio G, Elbourne DR, et al. Consort 2010 statement: extension to cluster randomised trials. BMJ 2012;345: e5661.

23. Torgerson DJ. Contamination in trials: is cluster randomisation the answer? BMJ 2001;322:355-7.
24. Pejtersen JH, Kristensen TS, Borg V, et al. The second version of the Copenhagen Psychosocial Questionnaire. Scand J Public Health 2010;38(3 Suppl):8-24.

25. Sions JM, Hicks GE. Fear-avoidance beliefs are associated with disability in older American adults with low back pain. Phys Ther 2011;91:525-34.

26. Caille A, Kerry S, Tavernier E, et al. Timeline cluster: a graphical tool to identify risk of bias in cluster randomised trials. BMJ 2016;354:i4291.

27. Rutterford C, Copas A, Eldridge S. Methods for sample size determination in cluster randomized trials. Int $\mathrm{J}$ Epidemiol 2015;44:1051-67.

28. Stephane Champely S, Ekstrom C, Dalgaard P, et al. Basic functions for power analysis. Secondary basic functions for power analysis 2015. https://cran.r-project.org/web/packages/pwr/pwr.pdf

29. Eldridge SM, Ashby D, Kerry S. Sample size for cluster randomized trials: effect of coefficient of variation of cluster size and analysis method. Int J Epidemiol 2006;35:1292-300.

30. van Breukelen GJ, Candel MJ. Calculating sample sizes for cluster randomized trials: we can keep it simple and efficient! J Clin Epidemiol 2012;65:1212-18.

31. Teare MD, Dimairo M, Shephard N, et al. Sample size requirements to estimate key design parameters from external pilot randomised controlled trials: a simulation study. Trials 2014;15:264.

32. Diaz-Ordaz K, Kenward MG, Cohen A, et al. Are missing data adequately handled in cluster randomised trials? A systematic review and guidelines. Clin Trials 2014:11:590-600.

33. Field A. Discovering statistics using R. 1 Ed: SAGE Publications Ltd, 2012.

34. Nielsen RO, Malisoux L, Moller M, et al. Shedding light on the etiology of sports injuries: a look behind the scenes of time-to-event analyses. J Orthop Sports Phys Ther 2016;46:300-11.

35. Spruance SL, Reid JE, Grace M, et al. Hazard ratio in clinical trials. Antimicrob Agents Chemother 2004;48:2787-92.

36. Recommendations for the conduct, reporting, editing and publication of scholarly work in medical journals. Secondary recommendations for the conduct, reporting, editing and publication of scholarly work in medical journals. http://www.icmje.org/icmje-recommendations.pdf 